\title{
Avanços na Análise de Fadiga de Compostos de Borracha
}

\author{
Renan Rodrigues de Mello Ozelo ${ }^{1}$, Paulo Sollero ${ }^{2}$, Argemiro Luis de Aragão Costa ${ }^{1}$ \\ ${ }^{1}$ Pirelli Pneus Ltda \\ ${ }^{2}$ DMC - FEM - UNICAMP
}

renan.ozelo@pirelli.com, sollero@fem.unicamp.br, argemiro.costa@pirelli.com

\section{RESUMO}

Este artigo tem como objetivo abordar o tema da fadiga em compostos de borracha, detalhar as teorias, equações e conceitos teóricos, destacando os recentes avanços. Pesquisas aplicadas e validações experimentais serão apresentadas de modo a exemplificar o uso dos métodos e técnicas adotadas. Em particular, será enfatizada a aplicação destas técnicas no desenvolvimento de pneus. Basicamente, a análise de falha pode ser separada em dois estágios: a nucleação e o crescimento de uma trinca. A mecânica do dano é a área de estudo responsável por analisar o estágio de nucleação da trinca, enquanto que a mecânica da fratura avalia a propagação da trinca até o colapso total da estrutura. A mecânica configuracional e a abordagem do plano crítico vêm sendo aplicadas nos últimos anos para análise de dano em compostos de borracha. Ambas as abordagens apresentam bons resultados na validação experimental considerando carregamentos multiaxiais de fadiga. Para simular a propagação da trinca em elastômeros existem alguns métodos numéricos para determinar o percurso de propagação na estrutura, exemplificados neste trabalho. Este tipo de análise visa identificar os pontos críticos na estrutura e evitar falhas catastróficas.

\section{INTRODUÇÃO}

O comportamento reológico dos compostos de borracha e sua capacidade de suportar altas deformações sem apresentar plasticidade ou ruptura, fazem com que este tipo de material seja ideal para aplicações, tais como: pneus, isoladores de vibração, correias e transmissão, amortecedores de impacto, artigos médicos, calçados, dentre outros.

Aplicados em conjunto com outros componentes estruturais, os compostos de borracha precisam ser submetidos a ensaios exaustivos de durabilidade e integridade antes de serem transformados em produtos pela indústria e chegarem ao consumidor final. A realização destes ensaios experimentais alonga de forma significativa o tempo e o custo de desenvolvimento de um produto, algumas vezes exigindo um grande número de protótipos.

A modelagem numérica tem auxiliado muito neste sentido, com o desenvolvimento de modelos preditivos capazes de entender comportamentos mecânicos, avaliar processabilidade, estimar a integridade estrutural e a vida em fadiga, dentre outras características essências para a produção e homologação de um novo produto. 
Do ponto de vista de durabilidade, integridade e vida em fadiga, a mecânica do dano e a mecânica da fratura são importantes áreas do conhecimento, que auxiliam na compreensão e na análise do desempenho dos materiais empregados como componentes estruturais, quando submetidos à carregamentos cíclicos. Porém, diferentemente dos modelos já consolidados, aplicados aos metais e suas ligas, os modelos de falha utilizados na análise de compostos de borracha são recentes e com muitos pontos ainda em aberto, desafiando cientistas e pesquisadores. Ainda não existe um modelo de falha consolidado para os elastômeros.

Basicamente, a análise de falha de um componente estrutural pode ser dividida em duas etapas: nucleação de uma trinca e propagação da trinca [1]. Atribui-se à mecânica do dano o estudo da primeira etapa, a nucleação da trinca, enquanto que a mecânica da fratura avalia a etapa de propagação da trinca até sua ruptura total. A seguir, discorreremos de forma breve sobre cada uma destas áreas de estudo.

A mecânica do dano tem como objetivo prever a nucleação de macro trincas, através da coalescência de micro defeitos e vacâncias, a fim de estimar a vida em fadiga de componentes estruturais [2]. Basicamente, a mecânica do dano avalia os históricos de tensão e deformação do componente e através de propriedades materiais obtidos experimentalmente, tais como a curva $\mathrm{S}$ $\mathrm{N}$, estima o tempo de vida do componente, para uma condição de trabalho pré-determinada.

Para a análise de elastômeros, abordagens convencionais, utilizando tensores de deformação e tensão e/ou a densidade de energia de deformação, mostram-se efetivos apenas em casos particulares, não obtendo sucesso em condições de carregamentos multiaxiais. Dessa forma, abordagens mais elaboradas vêm sendo desenvolvidas, visando avaliar o dano em condições de trabalho mais complexas.

O processo de fadiga multiaxial corresponde a uma configuração complexa de solicitações mecânicas que inclui os diversos tipos de deformações combinadas: tração, compressão, torção e cisalhamento. Solicitações deste tipo são comuns em componentes estruturais.

Considerando carregamentos multiaxiais, duas abordagens vêm obtendo bons resultados na análise da mecânica do dano em elastômeros. São estas: A mecânica configuracional e a abordagem do plano crítico. $\mathrm{Na}$ continuidade deste artigo serão apresentados os conceitos básicos e formulações de ambas as abordagens.

Em paralelo aos avanços da mecânica do dano, outra área importante na análise de integridade e fadiga é a mecânica da fratura. A mecânica da fratura de elastômeros vem sendo estudada desde 1953, quando Rivlin \& Thomas [3] introduziram o parâmetro $T$, denominado energia de ruptura ou energia de rasgamento (tearing energy). Porém, desde então, os maiores avanços nesta área se deram no âmbito experimental.

Atualmente, existem máquinas e dispositivos comerciais, suportados por normas técnicas, que auxiliam no levantamento das propriedades de fratura de compostos de borracha. No entanto, ainda é raro encontrar na indústria modelos e aplicações práticas pautadas na teoria da mecânica da fratura. Isto se deve, principalmente, pela dificuldade em modelar problemas deste tipo e efetiva aplicação dos parâmetros levantados experimentalmente. 
Técnicas de modelagem para problemas de propagação de trincas em estruturas vêm crescendo sensivelmente nos últimos anos e os métodos numéricos são fundamentais para tal crescimento. Um bom exemplo da aplicação dos métodos numéricos na análise de fratura é o XFEM (eXtended Finite Element Method), que se trata de uma extensão do método dos elementos finitos, desenvolvido especificamente para a análise de propagação de trincas. Contudo, tais aplicações muitas vezes são direcionadas e validadas para modelos lineares elásticos e elástoplásticos, ou seja, aplicados somente para materiais metálicos ou com comportamento mecânico semelhante.

Existe uma dificuldade especial em analisar problemas da mecânica da fratura em elastômeros. A teoria convencional, baseada em fatores de concentração de tensão, não se aplica para tais materiais. Para isto é necessário a utilização de teorias generalizadas baseadas em métodos de energia, como por exemplo, a Integral-J, proposta por Rice em 1968 [4]. Com todas estas dificuldades, a análise de fratura em elastômeros muitas vezes é deixada de lado. Fato que pode ter consequências catastróficas, como as ocorridas no anel de vedação do ônibus espacial Challenger em 1986 e no recall bilionário de cerca de 13,5 milhões de pneus em 2000, nos EUA.

A despeito destas duas grandes áreas de estudo na análise de integridade, outra abordagem vem ganhando força nos últimos anos. Esta abordagem é chamada de análise multiescala. A análise multiescala tem como característica analisar diversas escalas do material (macro escala, mesoescala, microescala, e nanoescala) a fim de solucionar os mais diferentes tipos de problemas. Nos últimos anos vem crescendo significativamente trabalhos com este enfoque e não é diferente quando falamos de análise de integridade. Uma das vantagens de tipo de abordagem na análise de compostos de borracha é o fato de ser possível modelar a interação molecular entre as cadeias poliméricas e assim propor mecanismos de falha que venham a caracterizar o dano macroscópico. Com a finalidade de exemplificar os avanços e a aplicação dos estudos nesta "nova" área do conhecimento, faremos no final de artigo um breve resumo do trabalho de Dal \& Kaliske [5], que propuseram um novo modelo constitutivo micromecânico acoplado com um mecanismo de falha.

\section{MECÂNICA DO DANO DE ELASTÔMEROS}

\subsection{Mecânica computacional}

A mecânica configuracional (configurational mechanics) é uma das abordagens que vem sendo desenvolvida nos últimos anos para a análise de dano em compostos de borracha $[6,7,8]$. Esta abordagem baseia-se na teoria geral proposta por Eshelby e utiliza o tensor de Eshelby $(\boldsymbol{\Sigma})$ como variável para determinar o dano no material.

O tensor de Eshelby é resultado da solução de um problema idealizado por ele, visando quantificar a variação da energia devido à mudança de posição de um defeito em um corpo carregado [9, 10]. Uma breve demonstração do problema proposto por Eshelby e o equacionamento aplicado no desenvolvimento do tensor, também é apresentado por Verron [6]. O tensor de Eshelby é definido pela seguinte equação:

$$
\boldsymbol{\Sigma}=W \mathbf{I}-\mathbf{F}^{T} \mathbf{P},
$$


na qual $W$ é a densidade de energia de deformação, I é o tensor identidade, $\mathbf{F}$ é o tensor gradiente de deformação e $\mathbf{P}$ é o primeiro tensor de tensões de Piola-Kirchhoff.

Reavaliando a formulação do tensor de Eshelby, de modo a expressá-lo em uma formulação Lagrangiana, tem-se a seguinte relação:

$$
\mathbf{P}=J \boldsymbol{\sigma} \mathbf{F}^{-T}
$$

onde $J$ é o Jacobiano do tensor de gradiente de deformação, ou seja, a deformação volumétrica e $\boldsymbol{\sigma}$ é o tensor de tensões de Cauchy. Substituindo a equação (2) em (1), obtém-se:

$$
\boldsymbol{\Sigma}=W \mathbf{I}-J \mathbf{F}^{T} \boldsymbol{\sigma} \mathbf{F}^{-T}
$$

Para elasticidade linear, na qual comumente se aplica o conceito de deformações infinitesimais, $\mathrm{Wu}$ [11] descreve sobre a possibilidade do tensor de Eshelby ser representado pela seguinte equação:

$$
\boldsymbol{\Sigma}=W \mathbf{I}-\boldsymbol{\sigma} \mathbf{F},
$$

ao passo que deformações lineares não fazem distinção entre coordenadas Lagrangianas e Eulerianas.

Supondo que a abertura e fechamento de micro defeitos e micro trincas se devem apenas às tensões normais e não devido às tensões de cisalhamento, tem-se o parâmetro de dano $\left(\Sigma^{*}\right)$ determinado a partir do valor absoluto do menor autovalor do tensor de Eshelby. O critério é então definido como:

$$
\Sigma^{*}=\left|\min _{i=1,2,3}\left[\Sigma_{i}, 0\right]\right|
$$

onde $\Sigma_{i}$ são as tensões principais do tensor. A operação $\min [\mathbf{\square}, 0]$ corresponde a seguinte relação:

$$
\begin{cases}\mathbf{\square} \geq 0 & \min [\mathbf{\square}, 0]=0 \\ \mathbf{\square}<0 & \min [\mathbf{\square}, 0]=\mathbf{\square}\end{cases}
$$

Esta operação garante que autovalores maiores de zero sejam desconsiderados. Dessa forma, os efeitos de fechamento das micro falhas não são considerados, por não causar o crescimento destes defeitos.

Verron [6] mostra em seu trabalho a capacidade do parâmetro $\Sigma^{*}$ em quantificar a taxa de liberação e energia em pequenos defeitos. Além disso, pontua que o acoplamento deste parâmetro com um modelo viscoelástico tem sido aplicado com sucesso ao prever a vida em fadiga de compostos de borracha natural em condições de carregamentos cíclicos.

A vida em fadiga é determinada através da seguinte equação: 


$$
N_{f}=\left(\frac{\Sigma^{*}}{\Sigma_{0}}\right)^{\beta}
$$

na qual o parâmetro $\Sigma^{*}$ é correlacionado com a tenacidade e a fadiga do material, representada pelos parâmetros materiais $\Sigma_{0}$ e $\beta$, obtidos experimentalmente a partir de ensaios de fadiga uniaxial.

\subsection{Abordagem do plano crítico}

A abordagem do plano crítico (critical plane approach), também aplicada para a análise e dano, foi proposta por Saintier et al. [8] e, do mesmo modo que a abordagem da mecânica configuracional, apresenta resultados práticos interessantes.

Nesta abordagem são considerados dois mecanismos, o dano e a cristalização, este último denominado na formulação como reforço. Obtém-se então uma tensão equivalente $(\Phi)$, que é utilizada para determinar a vida em fadiga do material.

A tensão equivalente é definida da seguinte forma:

$$
\Phi=\frac{\Phi_{\text {dano }}}{1+\Phi_{\text {reforço }}},
$$

na qual $\Phi_{\text {dano }}$ (possui unidades de tensão) e $\Phi_{\text {reforço (adimensional) são parâmetros avaliados a }}$ partir do histórico de tensões. A contribuição do dano é equivalente à máxima tensão principal de Cauchy suportado pelo material em um ciclo:

$$
\Phi_{\text {dano }}=\max _{t=0, t_{c}}\left\{\max _{i=1,2,3}\left[\sigma_{i}(t)\right]\right\}=\sigma_{i_{\max }}\left(t_{\max }\right),
$$

onde $t_{c}$ é o tempo de duração do ciclo, $\sigma_{i}(t)$ são as tensões principais do tensor de Cauchy no tempo $t$ e $t_{\max }$ corresponde ao tempo no qual a mais alta tensão principal é alcançada durante todo o ciclo. Além disso, a máxima tensão principal no tempo $t_{\text {max }}$ faz com que seja definido o plano crítico $\mathbf{n}_{\mathrm{CP}}\left(t_{\max }\right)$, que corresponde ao plano normal à direção associada ao tensor $\sigma_{i}\left(t_{\max }\right)$. Contudo, para a análise completa de um ciclo de fadiga, é necessário mapear a posição do plano crítico em cada espaço de tempo analisado. E para isso, primeiro deve-se obter a posição do plano na configuração de referência (condição não deformada),

$$
\mathbf{N}_{\mathrm{CP}}=\mathbf{n}_{\mathrm{CP}}(t=0)=\frac{\mathbf{F}^{\mathrm{T}}\left(t_{\max }\right) \mathbf{n}_{\mathrm{CP}}\left(t_{\mathrm{max}}\right)}{\left\|\mathbf{F}^{\mathrm{T}}\left(t_{\max }\right) \mathbf{n}_{\mathrm{CP}}\left(t_{\max }\right)\right\|} .
$$

Dessa forma, a posição do plano crítico em um tempo qualquer é dada por:

$$
\mathbf{n}_{\mathrm{CP}}(t)=\frac{\mathbf{F}^{-\mathrm{T}}(t) \mathbf{N}_{\mathrm{CP}}}{\left\|\mathbf{F}^{-\mathrm{T}}(t) \mathbf{N}_{\mathrm{CP}}\right\|} .
$$


Saintier et al. [8] considera o parâmetro de reforço $\Phi_{\text {reforço }}$ sendo diretamente proporcional ao processo de cristalização induzida pela deformação, caracterizada pela cristalinidade $X_{\mathrm{c}}$ e um parâmetro material $\xi$, ou seja,

$$
\Phi_{\text {reforço }}=(1-\xi) X_{\mathrm{c}}
$$

Para a condição de $X_{\mathrm{c}}$ igual à zero, tem-se o material totalmente amorfo. Dessa forma, a tensão equivalente $\Phi$ equivale somente ao parâmetro de dano. A relação empírica aplicada por Saintier entre $X_{\mathrm{c}}$ e o tensor de Cauchy é dada a seguir:

$$
X_{\mathrm{c}}\left(\sigma_{\text {reforço }}\right)=0,3\left[1-\exp \left(-D \cdot\left\langle\sigma_{\text {reforço }}-\sigma_{\text {limiar }}\right\rangle\right)\right] \text {, }
$$

onde $\sigma_{\text {reforço }}$ corresponde à tensão de reforço, $\sigma_{\text {limiar }}$ é o limiar da tensão de cristalização, $D$ é um parâmetro material e o operador $\langle\boldsymbol{\square}\rangle$ (MacCauley bracket) é definida por:

$$
\langle\boldsymbol{\square}\rangle= \begin{cases}\boldsymbol{\square} & \text { se } \boldsymbol{\square}>0 \\ 0 & \text { se } \boldsymbol{\square} \leq 0\end{cases}
$$

O valor constante de 0,3 remete à máxima cristalização observada na borracha natural.

A determinação da tensão de reforço é obtida a partir da tensão normal $\sigma_{n}$ e de cisalhamento $\tau_{s}$ no plano crítico. Porém, primeiro é necessário definir a condição de solicitação mecânica existente para o cálculo da tensão de reforço:

- Se a menor tensão normal no plano crítico durante um ciclo for maior que zero, ou seja, $\sigma_{n}\left(t_{\min }\right)>0$. Então, a tensão de reforço é dada por:

$$
\sigma_{\text {reforço }}=\sqrt{\left[\sigma_{n}\left(t_{\min }\right)\right]^{2}-\left[\tau_{s}\left(t_{\min }\right)\right]^{2}} \text {. }
$$

- Se a menor tensão normal no plano crítico durante um ciclo for menor ou igual que zero, ou seja, $\sigma_{n}\left(t_{\min }\right) \leq 0$. Então, a tensão de reforço é dada por:

$$
\sigma_{\text {reforço }}=\tau_{s}\left(t_{\mathrm{cls}}\right) \text {, }
$$

onde $t_{\mathrm{cls}}$ determina o tempo de fechamento das microtrincas (vide Figura 1).

A tensão normal e tensão de cisalhamento no plano crítico são determinas respectivamente da seguinte forma:

$$
\begin{gathered}
\sigma_{n}(t)=\left[\sigma(t) \cdot \mathbf{n}_{\mathrm{CP}}(t)\right] \cdot \mathbf{n}_{\mathrm{CP}}(t) ; \\
\tau_{s}(t)=\sqrt{\left\|\sigma_{n}(t) \cdot \mathbf{n}_{\mathrm{CP}}(t)\right\|^{2}-\left[\sigma_{n}(t)\right]^{2}} .
\end{gathered}
$$

Como na abordagem anterior, a vida em fadiga do material é obtida a partir de uma função de lei de potências, que correlaciona a tensão equivalente com a tenacidade à fadiga do material: 


$$
N_{f}=\left(\frac{\Phi}{\Phi_{0}}\right)^{\alpha},
$$

onde $\Phi_{0}$ e $\alpha$ são parâmetros materiais obtidos experimentalmente, que representam a tenacidade a fadiga do material.

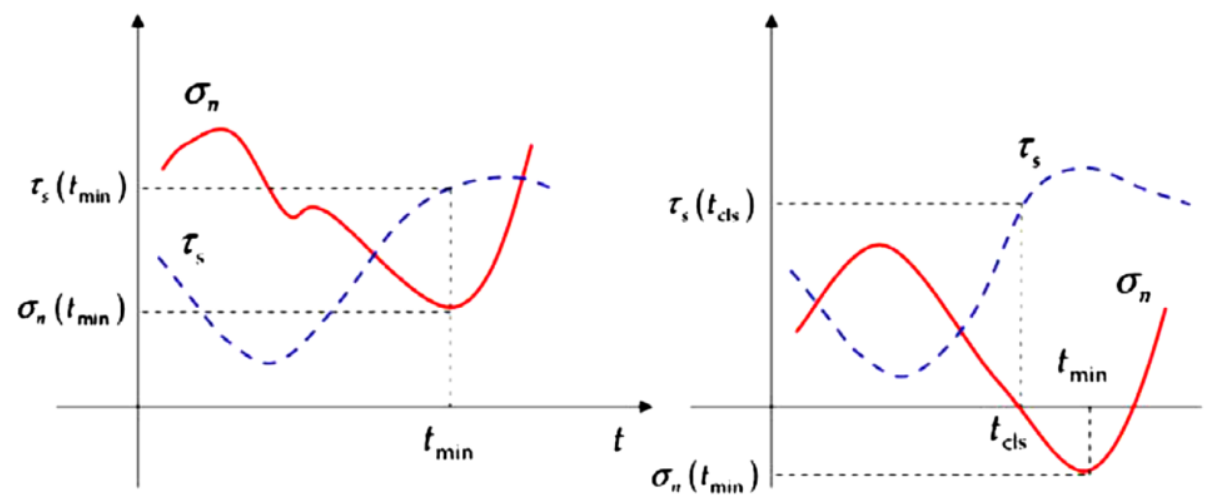

Figura 1 - Evolução arbitrária da tensão normal e tensão de cisalhamento no plano crítico durante um ciclo [9].

\subsection{Comparação com resultados experimentais}

Uma análise comparativa entre as duas abordagens apresentas e resultados experimentais é realizada no trabalho de Andriyana et al. [9]. De modo a obter a eficiência de cada uma das abordagens na previsão da vida em fadiga considerando condições de carregamento multiaxiais, foram testados diversos tipos de solicitações mecânicas cíclicas: torção simples, torção alternada, compressão/tração proporcional e tração/tração. Para cada uma das solicitações mecânicas citadas foram testadas diversas condições de carga.

A Figura 2 mostra os resultados obtidos pelos autores na comparação entre a abordagem do plano crítico e a abordagem da mecânica configuracional.
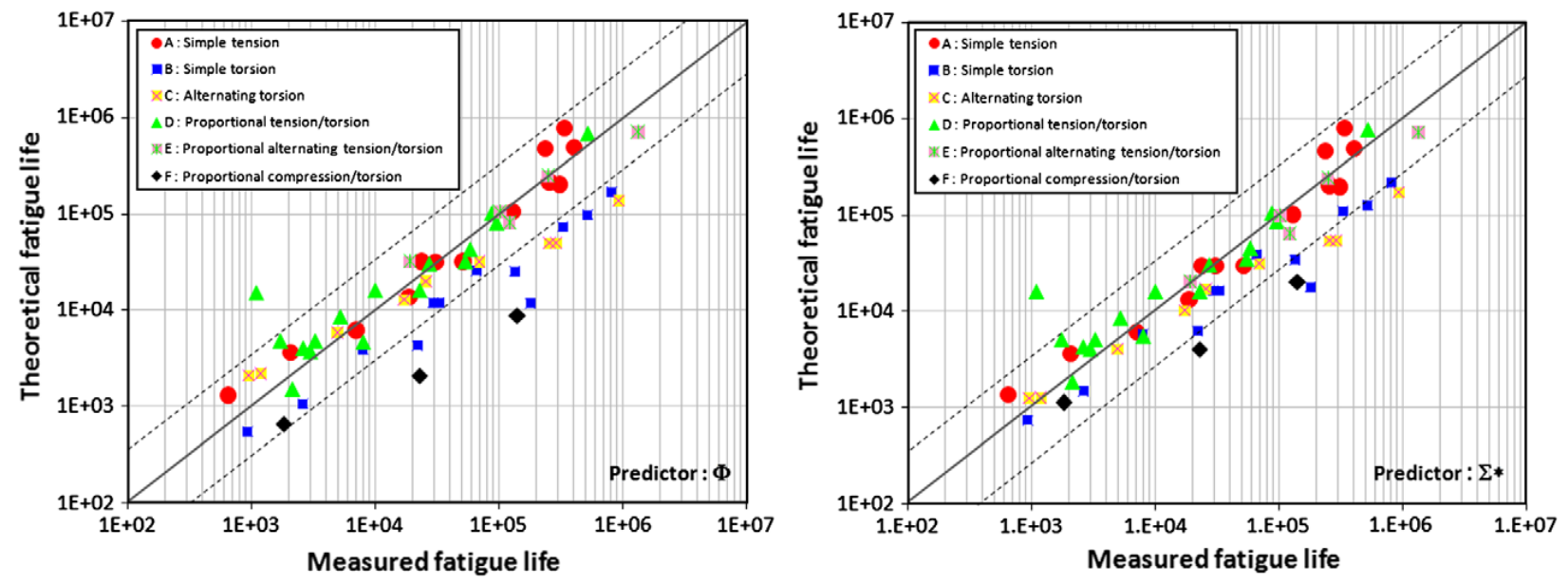

Figura 2 - Comparação entre os resultados teóricos e experimentais de vida em fadiga para as abordagens do plano crítico (esquerda) e da mecânica configuracional (direita) [9]. 
Conforme concluído pelos autores, ambas as abordagens apresentaram bons resultados na previsão da vida em fadiga. Maiores discrepâncias são observadas em condições nas quais a solicitação mecânica predominante é a torção. Na comparação entre as abordagens testadas, a abordagem da mecânica configuracional apresentou melhores resultados.

\subsection{Método de análise de dano e fadiga}

Para a construção de um método de análise de dano e fadiga, optou-se inicialmente pela abordagem da mecânica configuracional. A aplicação desta abordagem se deu com a utilização do método dos elementos finitos (MEF) através do software ABAQUS ${ }^{\circledR}$. Andriyana et al. [9] discorrem sobre o fato da implementação desta abordagem no MEF, particularmente na avaliação do ciclo de fadiga, ainda está sendo investigada. É importante salientar, que será apresentado a seguir uma implementação de um método de análise por elementos finitos (FEA), ainda em desenvolvimento, passível de validação, que considera somente a condição de máxima carga do ciclo de carregamento para a estimativa da vida em fadiga.

O software ABAQUS ${ }^{\circledR}$ foi utilizado para a solução do modelo em elementos finitos. Para a análise de dano e fadiga foram desenvolvidas rotinas nas linguagens de programação FORTRAN e PYTHON para a obtenção dos tensores gradiente de deformação e de Eshelby, respectivamente. Além de obter o tensor de Eshelby, a rotina em PYTHON é responsável pelos cálculos do parâmetro de dano $\Sigma^{*}$, dos vetores normais referentes ao plano de propagação da trinca e da vida em fadiga do modelo. Na Figura 3 é apresentado o fluxo de análise de fadiga com as rotinas desenvolvidas.

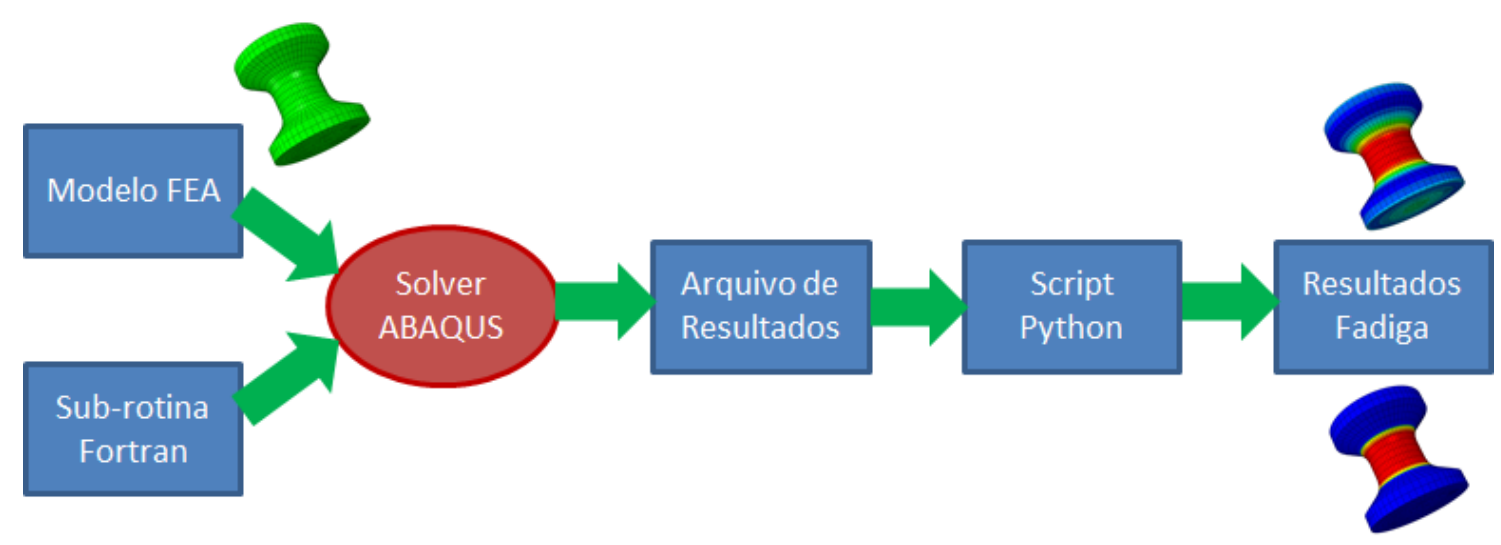

Figura 3 - Fluxograma da análise de dano e fadiga.

Considerando os dados geométricos (vide Figura 4) e as propriedades materiais e de fadiga dos corpos de prova utilizados nos trabalhos de Saintier et al. [8] e Andriyana et al. [9], foi realizada uma primeira avaliação do método de análise. $\mathrm{O}$ modelo hiperelástico aplicado para caracterização do comportamento mecânico do composto de borracha foi o modelo NeoHookeano definido pela seguinte função densidade de energia de deformação:

$$
W=C_{10}\left(I_{1}-3\right)+\frac{1}{D_{1}}(J-1)^{2},
$$


onde $C_{10}$ e $D_{1}$ são propriedades do material, $I_{1}$ é o primeiro invariante de deformação e $J$ corresponde à deformação volumétrica. $\mathrm{O}$ valor da constante $C_{10}=0,36 \mathrm{MPa}$ foi obtida por Andriyana et al. [9] através do ensaio experimental uniaxial do material. Já a constante $D_{1}=$ 0,002 foi definido devido à incompressibilidade do material. Os parâmetros de dano alimentam a rotina em PYTHON para a análise de fadiga. As constantes da Equação (7) também foram determinadas no trabalho de Andriyana et al. [9] de forma experimental. Os valores obtidos foram $\Sigma_{0}=59,0 \mathrm{MJ} / \mathrm{m}^{3}$ e $\beta=-3,18$. A obtenção destas constantes fornece uma curva de fadiga tipo S-N, mostrada na Figura 5 , que vincula o parâmetro de dano $\Sigma^{*}$ com o número de ciclos de fadiga do material.

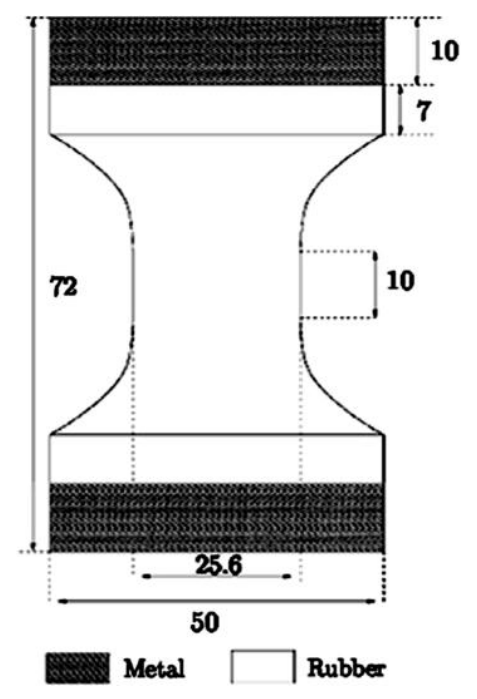

Figura 4 - Geometria do corpo de prova usado nos ensaios de fadiga por Saintier et al. [8].

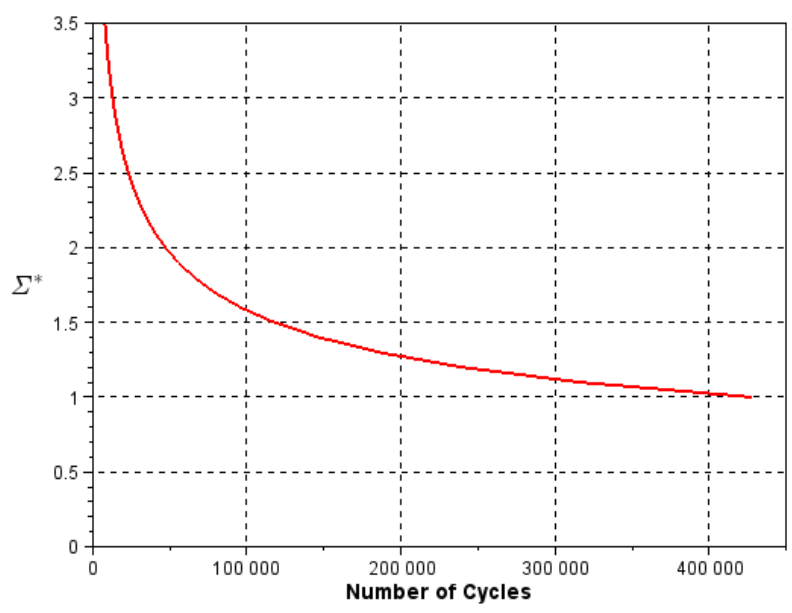

Figura 5 - Curva do parâmetro de dano pelo número de ciclos.

O método permite obter o campo escalar do parâmetro de dano $\Sigma^{*}$ (Figura 6), o campo vetorial que define os planos de nucleação das trincas (Figura 7), além de um campo escalar que determina a vida em fadiga (Figura 8) de cada elemento do flexível. 

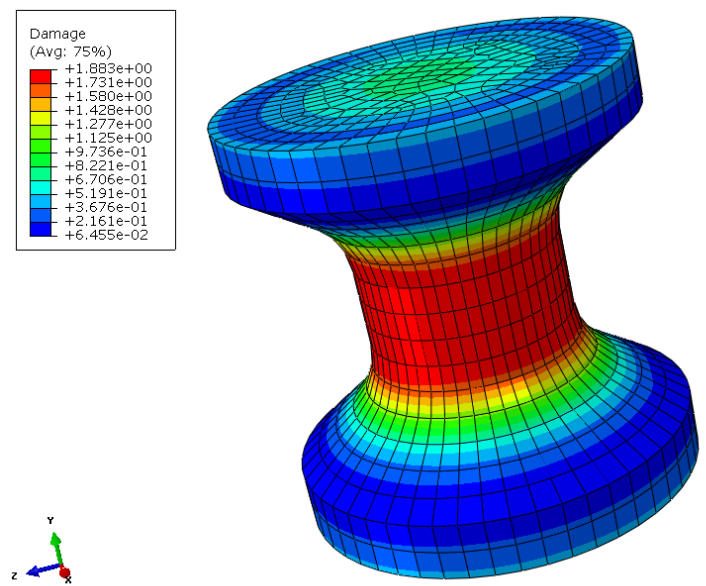

Figura 6 - Campo escalar do parâmetro de dano $\Sigma^{*}$.

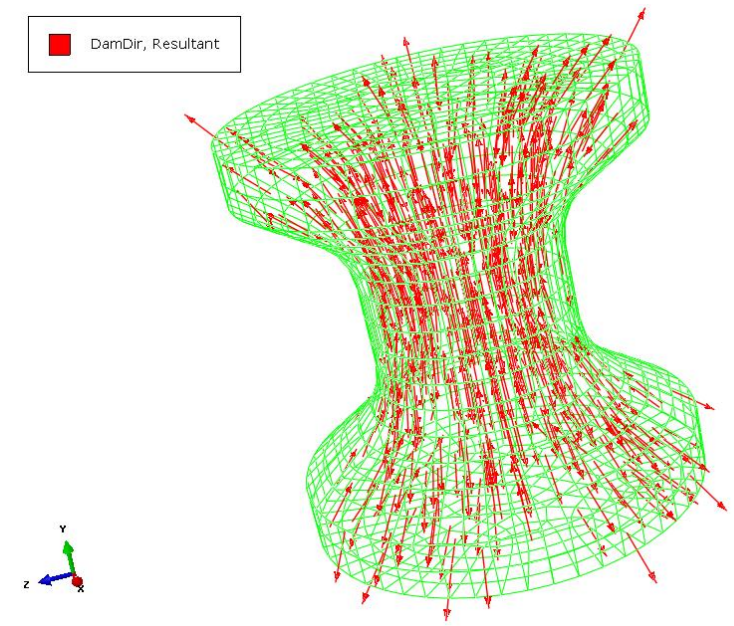

Figura 7 - Campo vetorial dos planos de nucleação da trinca.
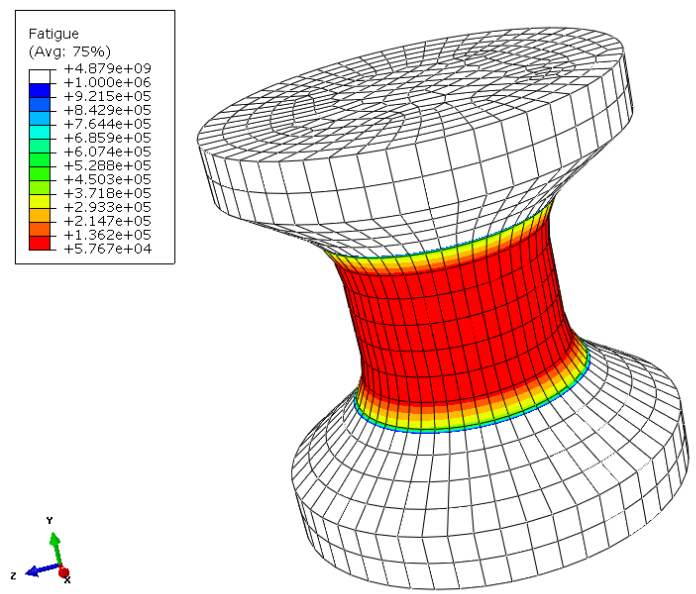

Figura 8 - Campo escalar da vida em fadiga (número de ciclos). 


\subsection{Aplicação do método de análise de dano e fadiga}

Apesar da aplicação da análise de fadiga no desenvolvimento do produto estar pendente da validação experimental do método, os resultados obtidos são promissores e coerentes com o que se observa no produto.

A estrutura de um pneumático é um compósito formado por diversos materiais (elastômeros, têxteis, metais) com diferentes módulos de elasticidade e comportamento mecânico (vide Figura 9). Regiões com grandes gradientes de deformação são comuns neste tipo de estrutura e a nucleação de trincas é inevitável. Neste contexto, utiliza-se a análise da mecânica da fratura para evitar que as trincas macroscópicas nucleadas se propaguem, podendo causar a falha catastrófica da estrutura.

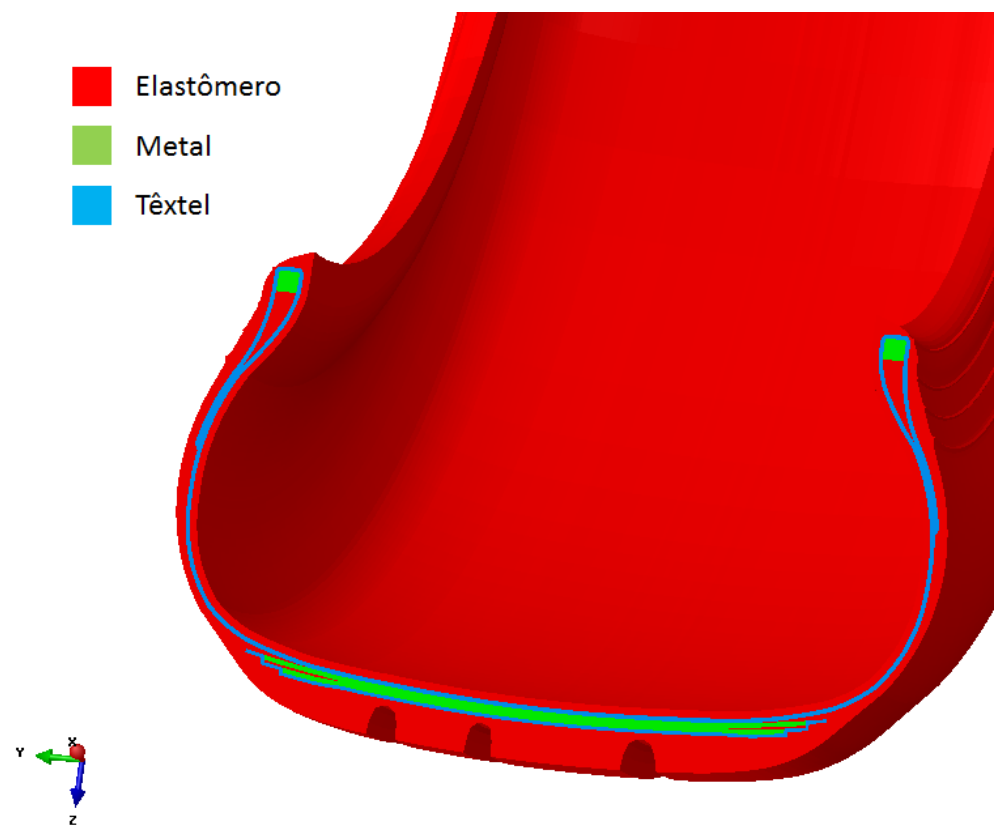

Figura 9 - Estrutura de um pneumático.

A análise de dano no pneu foi capaz de mapear regiões como extremidades de cinturas e frisos metálicos (Figura 10). Estas regiões possuem grande probabilidade de nucleação de trincas, pois se tratam de zonas de interface metal/elastômero, que apresentam grandes concentrações de tensões. A análise de vida em fadiga ainda não foi possível, pois ainda é necessária a validação do método e caracterização das propriedades de fadiga de cada um dos compostos de borracha presente nesta estrutura.

\section{MECÂNICA DA FRATURA DE ELASTÔMEROS}

No âmbito da mecânica da fratura aplicada a elastômeros, os últimos avanços remetem a aplicação de métodos capazes de determinar o percurso de propagação de trincas. Este tipo de análise, muito utilizada em indústrias de óleo \& gás, naval, aeronáutica, dentre outras, tem como objetivo identificar pontos críticos na estrutura e evitar falhas catastróficas. 


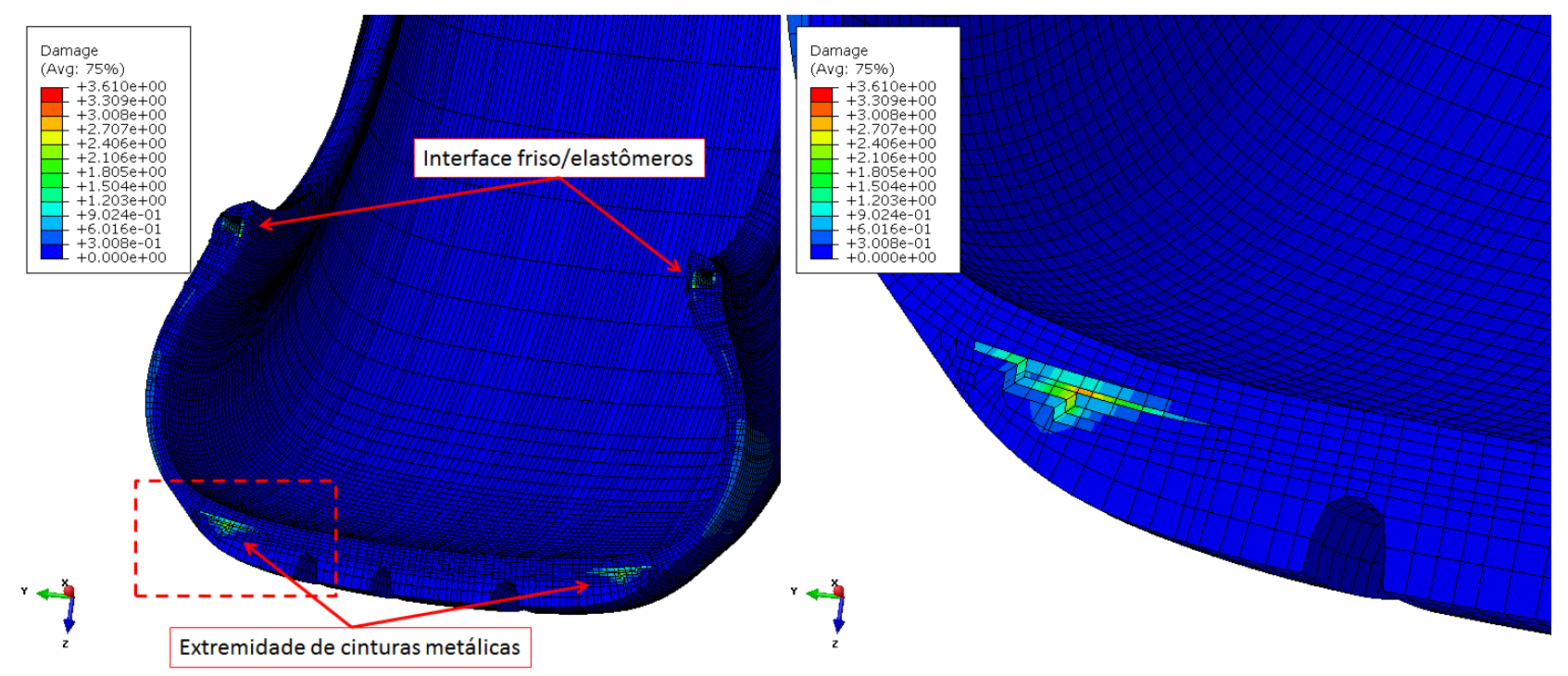

Figura 10 - Análise de dano na estrutura de um pneumático.

Para a modelagem de problemas de propagações de trincas em compostos de borracha, alguns trabalhos propõem métodos para determinar o caminho percorrido por uma trinca sob uma estrutura, dada uma solicitação mecânica. Dentre os trabalhos publicados, destacam-se os trabalhos de Geubelle \& Knauss [10], Loppin [11] e Ozelo et al. [12].

No trabalho de Ozelo et al. [12] é proposta uma técnica alternativa para avaliar o percurso de propagação da trinca baseada na Integral-J e no princípio da mínima energia potencial total.

A Integral-J corresponde à variação de energia interna de um corpo devido à propagação de uma trinca e é definida pela seguinte equação:

$$
\mathcal{J}=\int_{\Gamma_{0}}\left(W n_{1}-T_{i} \frac{\partial u_{i}}{\partial x_{1}}\right) \mathrm{d} \Gamma_{0}
$$

na qual $\Gamma_{0}$ é um contorno predefinido no corpo, $T_{i}$ e $u_{i}$ são, respectivamente, as forças de superfície e os deslocamentos prescritas no contorno $\Gamma_{0}$ e $n_{1}$ é a projeção na direção $X_{1}$ do vetor normal ao contorno $\Gamma_{0}$ (ver Figura 11). A técnica resume-se, basicamente, em avaliar a Integral-J nas diversas direções possíveis de propagação da trinca, de modo a determinar a direção de máxima variação de energia devido à propagação, visando minimizar a energia interna do corpo. Para isto, devemos generalizar a Equação (21), introduzindo o vetor unitário $q_{i}$, definido por,

$$
q_{i}=\left\{\begin{array}{c}
\cos \theta \\
\operatorname{sen} \theta
\end{array}\right\}
$$

que determina a direção de propagação na qual a Integral-J será avaliada. A generalização bidimensional da Equação (21) é dada a seguir: 


$$
\mathcal{J}(\theta)=\int_{\Gamma_{0}}\left[W\left(n_{i} \cdot q_{i}\right)-T_{i}\left(q_{i} \cdot \nabla u_{i}\right)\right] \mathrm{d} \Gamma_{0},
$$

onde $\theta$ corresponde ao ângulo de propagação da trinca. Uma vez generalizada a Integral-J, a técnica propõe que a direção de propagação $\theta_{p}$ equivale ao máximo valor de $\mathcal{J}(\theta)$, ou seja,

$$
\mathcal{J}\left(\theta_{p}\right)=\max [\mathcal{J}(\theta)] .
$$

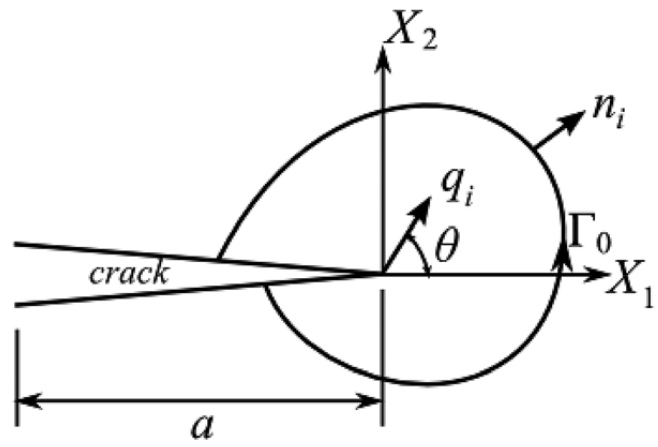

Figura 11 - Contorno fechado na ponta de uma trinca [12].

A técnica foi validada através de ensaios experimentais com corpos de provas com geometria que propiciam a mudança de direção da trinca. Os resultados são apresentados na Figura 12.

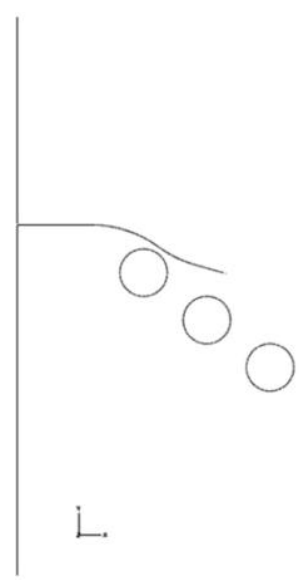

(a)

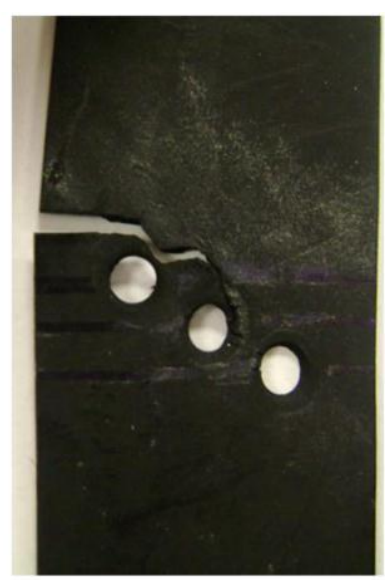

(b)

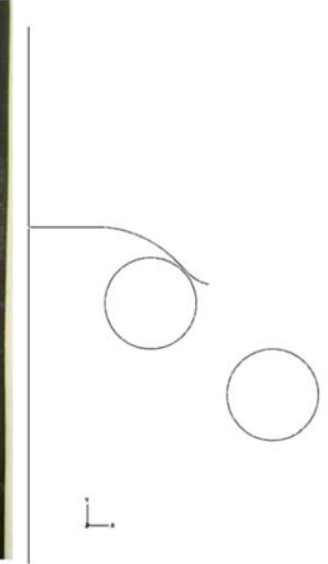

(a)

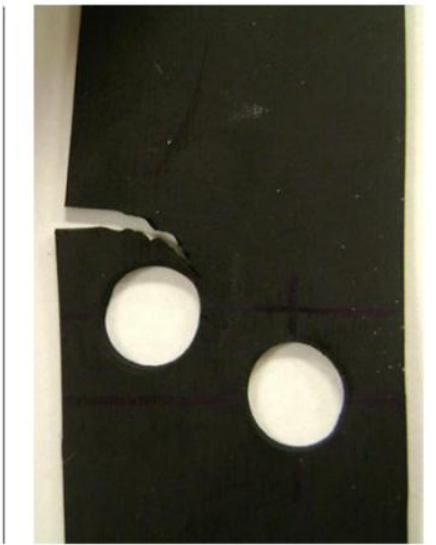

(b)

Figura 12 - Comparação entre resultados numéricos (a) e experimentais (b) para os corpos de prova com três furos (esquerda) e com dois furos (direita) [12].

\subsection{Aplicação da técnica de propagação de trinca}

A aplicação da técnica de propagação de trinca se deu na tentativa de caracterizar o percurso de propagação de uma trinca intencional na estrutura de pneu, de modo a otimizar a resistência banda de rodagem contra cortes acidentais, como por exemplo, solo com pedras ou no contato com objetos cortantes. 
Utilizou-se o MEF aplicando o conceito de análise global-local, mostrada na Figura 13, para avaliar a região de propagação. A comparação entre os resultados numérico e experimental, apresentada na Figura 14, demonstra que a técnica é capaz de determinar de forma satisfatória o percurso de propagação de uma trinca inclusive em estruturas complexas com solicitação mecânicas multiaxiais. A única restrição desta aplicação se deve ao fato de que ainda não é possível avaliar a propagação da trinca entre dois compostos de borracha. É importante ressaltar, que esta não é uma limitação da técnica, mas sim do modo como a técnica foi implementada no software ABAQUS ${ }^{\circledR}$.

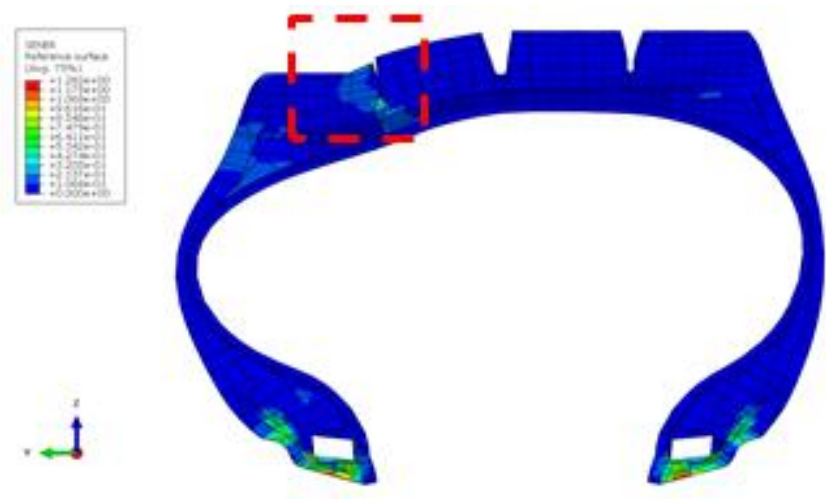

(a)

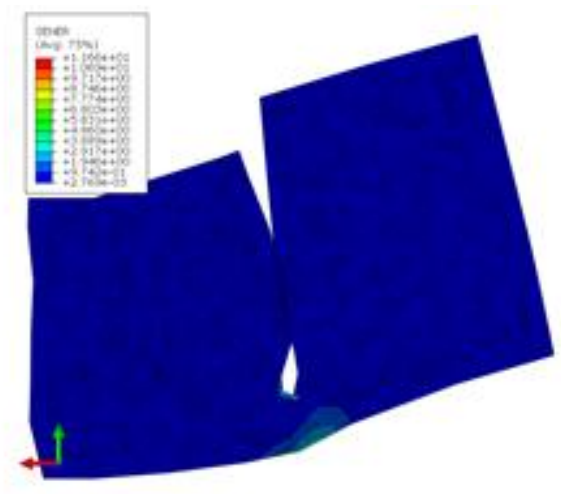

(b)

Figura 13 - Análise global-local. (a) Modelo global; (b) Modelo local.

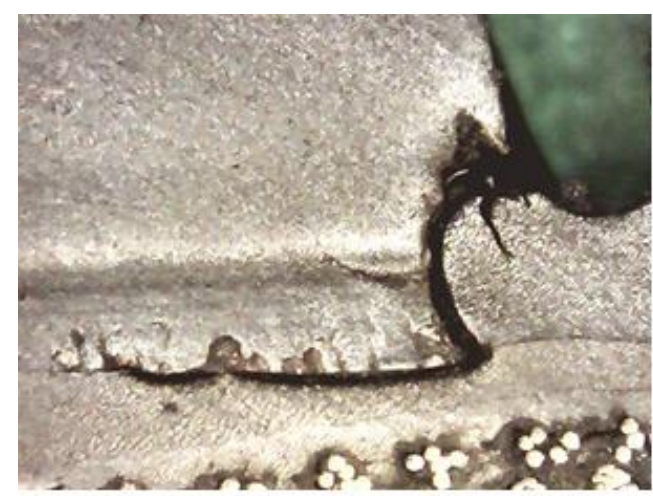

(a)

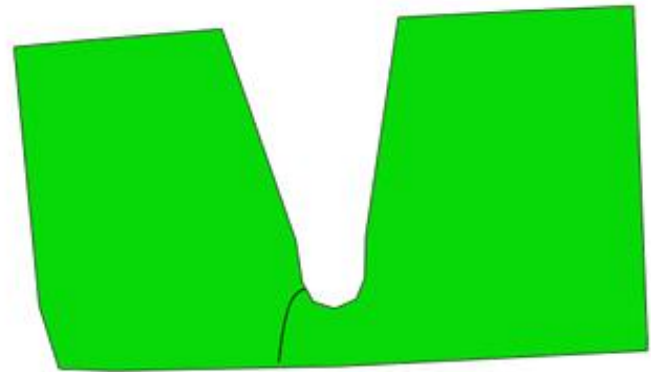

(b)

Figura 14 - Comparação entre os resultados numérico e experimental.

\section{ANÁLISE MULTIESCALA}

Como descrito anteriormente, a análise multiescala é uma das recentes áreas que vem sendo utilizada no estudo de integridade de compostos de borracha. Um exemplo de aplicação da multiescala na análise de integridade em elastômeros e a utilização do modelo proposto por Dal \& Kaliske [5] que possibilita caracterizar, via simulações computacionais, os limites de resistência mecânica dos materiais. 
Através de modelos micromecânicos, Dal \& Kaliske propuseram um modelo reológico capaz de avaliar a integridade do material através de modelos de mecânica estatística que visam caracterizar o comportamento das macromoléculas na microestrutura do material.

Neste trabalho, o rompimento da macromolécula é modelado a partir do potencial de Morse, no qual se deriva a micro força responsável pela quebra da cadeia polimérica, vide Figura 15.

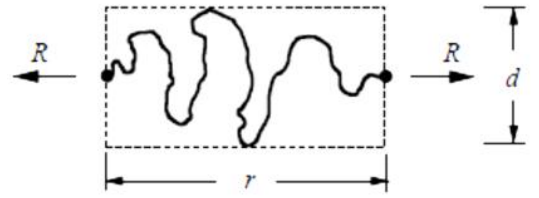

(A)

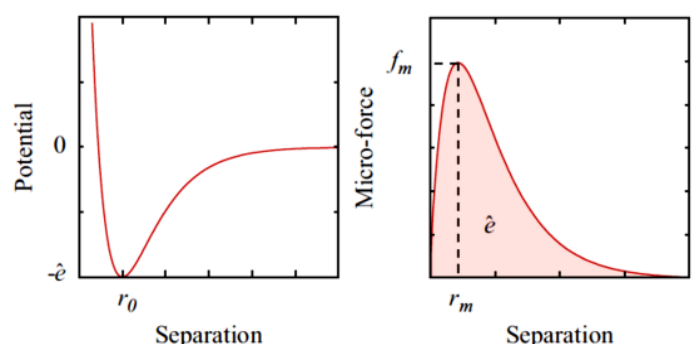

(B)

Figura 15 - (A) Representação geométrica de uma macromolécula; (B) Potencial de Morse e micro força de separação [5].

Desta forma, os autores desenvolveram um modelo constitutivo acoplado com um mecanismo de falha, capaz de limitar a energia absorvida pelo material. Na Figura 16 é feita a comparação do modelo descrito com modelos constitutivos que não apresentam tal mecanismo.
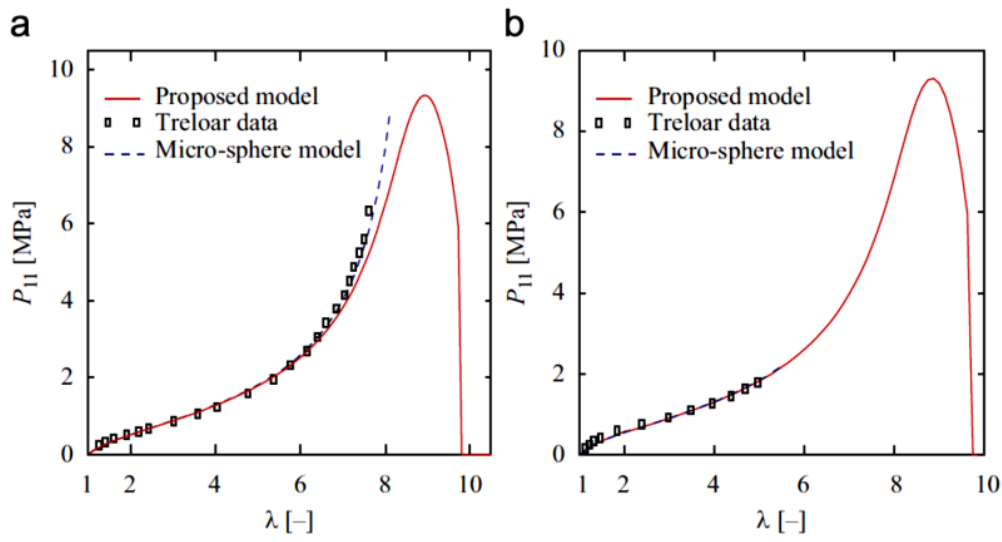

Figura 16 - Relação tensão-deformação (primeiro tensor de Piola-Kirchhoff vs. Alongamento).

(A) Ensaio uniaxial de tração; (B) Ensaio de cisalhamento puro [5].

No mesmo estudo, os autores avaliam também a mudança do comportamento mecânico do material devido ao envelhecimento induzido. Para isto, os autores partem do pressuposto que o envelhecimento pode ser representado basicamente pelo aumento do número de ligações cruzadas na microestrutura do composto de borracha, vide Figura 17. A validação da modelagem com dados experimentais é apresentada na Figura 18. 
a

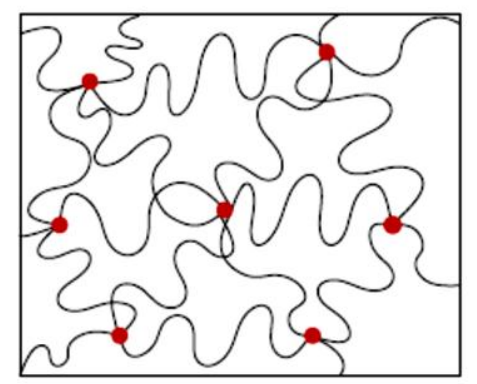

b

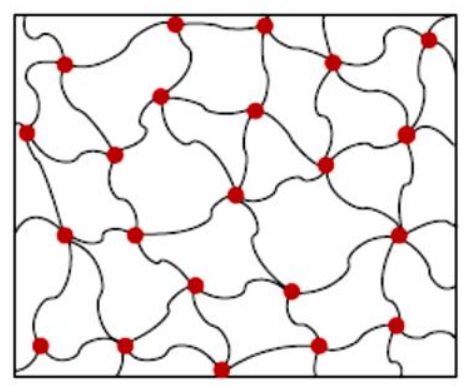

Figura 17 - Representação do processo de envelhecimento sob as ligações cruzadas na microestrutura de um composto de borracha. (A) Antes do envelhecimento; (B) Após o envelhecimento [5].

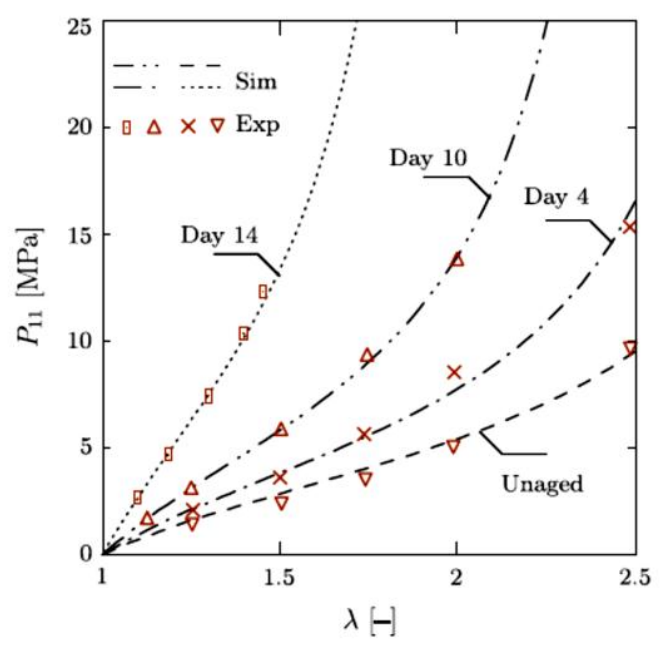

Figura 18 - Validação da modelagem de envelhecimento [5].

\section{CONCLUSÕES}

Os recentes avanços nas áreas da mecânica do dano e mecânica da fratura de elastômeros vêm de encontro à crescente solicitação das indústrias deste ramo, que é a política de redução de protótipos. Isso só é possível através da aplicação de técnicas confiáveis capazes de fornecer aos engenheiros informações sobre o comportamento do produto, que possibilitam ações corretivas e de melhoramento. Ainda é necessário consolidar grande parte das abordagens de dano e fratura aplicadas aos elastômeros, porém os avanços dos últimos anos são sensíveis e já possibilitam identificar e mapear regiões críticas na estrutura, como pode ser visto nas aplicações das técnicas contempladas neste trabalho.

A análise multiescala há muitos anos vem sendo estudada e aplicada na engenharia de um modo geral. Recentemente ganhou força, principalmente devido ao aumento da capacidade computacional. Os últimos avanços na área dos elastômeros remetem a modelos constitutivos 
mais complexos, que possibilitam obter respostas mais realísticas sobre comportamento dos materiais. Esta é uma tendência e promete grandes avanços para os próximos anos.

\section{REFERÊNCIAS}

[1] MARS, W. V., FATEMI, A., A literature survey on fatigue analysis approaches for rubber, International Journal of Fatigue, vol. 24, pp. 949-961, 2002.

[2] KACHANOV, L. M., Time of the rupture process under creep conditions, Izv. Akad. Nauk. S.S.R. Otd. Tech. Nauk., vol. 8, pp. 26-31, 1958.

[3] RIVLIN, R. S., THOMAS, A. G., Rupture of rubber I. Characteristic energy for tearing," Journal of Polymer Science, vol. 10, pp. 291-318, 1953.

[4] RICE, J. R., A path independent integral and the approximate analysis of concentration by notches and cracks, Journal of Applied Mechanics, vol. 35, pp. 379-386, 1968.

[5] DAL, H., KALISKE, M., A micro-continuum-mechanical material model for failure of rubber-like materials: Application to ageing-induced fracturing, Journal of the Mechanics and Physics of Solids, vol. 57, pp. 1340-1356, 2009.

[6] ANDRYIANA, A., SAINTIER, N., VERRON, E., Configurational mechanics and critical plane approach: Concept and application to fatigue failure analysis of rubberlike materials, International Journal of Fatigue, vol. 32, pp. 1627-1638, 2010.

[7] VERRON, E., Configurational mechanics: A tool to investigate fracture and fatigue of rubber, Rubber Chemistry and Technology, vol. 83, pp. 270-281, 2010.

[8] VERRON, E., ANDRIAYA, A., Definition of a new predictor for multiaxial fatigue crack nucleation in rubber, Journal of the Mechanics and Physics of Solids, vol. 56, pp. 417443, 2008.

[9] ESHELBY, J. D., The elastic energy-momentum tensor, Journal of Elasticity, vol. 5, n. 34, pp. 321-335, 1957.

[10] ESHELBY, J. D., The force on an elastic singularity, Philos Trans R Soc Lond, vol. 244, pp. 87-112, 1951.

[11] WU, C. H., The role of Eshelby stress in composition-generated and stress-assisted difusion, Journal of the Mechanics and Physics of Solids, vol. 49, pp. 1771-1794, 2001.

[12] SAINTIER, N., CAILlETAUD, G., PIQUES, R., Multiaxial fatigue life prediction for a natural rubber, International Journal of Fatigue, vol. 28, pp. 530-539, 2006. 
[13] GEUBELlE, P. H., KNAUSS, W. G., Finite strain at the tip of a crack in a sheet of hyperelastic material: I. Homogeneous case, Journal of Elasticity, vol. 35, pp. 61-98, 1994.

[14] LOPPIN, G., Plane Crack Propagation in a Hyperelastic Incompressible Material, International Journal of Fracture, vol. 109, pp. 169-188, 2001.

[15] OZELO, R. R. M., SOLLERO, P., COSTA, A. L. A., An alternative technique to evaluate crack propagation path in hyperelastic materials, Tire Science and Technology, vol. 40, pp. 42-58, 2012. 